\title{
Tree and shrub flora in the surroundings of the Parque Nacional do Iguaçu, Paraná State, Brazil: contribution to ecological restoration
}

\author{
Roque Cielo-Filho ${ }^{1,4}$, Geraldo Antonio Daher Corrêa Franco ${ }^{2}$, Fernando Periotto ${ }^{3}$, Osny Tadeu de Aguiar ${ }^{2}$, \\ João Batista Baitello ${ }^{2}$, Carla Daniela Câmara ${ }^{3}$, Carolina Rodrigues Sousa ${ }^{2}$ and Juliana Menezes de Jesus ${ }^{2}$
}

Received: 17.11.2016; accepted: 3.07.2017

\begin{abstract}
Tree and shrub flora in the surroundings of the Parque Nacional do Iguaçu, Paraná State, Brazil: contribution to ecological restoration). Aiming to expand the regional floristic knowledge and compile species information to subsidize restoration projects, we conducted a floristic survey in native forest fragments of an area located between the Parque Nacional do Iguaçu and the Área de Preservação Permanente do Lago de Itaipu, in western Paraná State. We found 204 species and 51 families, being the richest Fabaceae (29 spp.), Myrtaceae (18 spp.), Solanaceae (10 spp.), Euphorbiaceae, Meliaceae and Rutaceae (nine spp. each). The zoochoric syndrome was the most common ( $69 \%$ of the species), followed by anemochoric $(17 \%)$ and autochoric (14\%). The non-pioneer species were the most frequent (64\%). In general terms, these results agree qualitatively with those observed in seasonal semideciduous forests. Based on phytosociological data from other studies, we indicated 34 locally abundant species that can be used in greater proportions in restoration projects. We recorded seven species threatened and 15 exotic, of which 12 have invasive potential and require actions for controlling.
\end{abstract}

Keywords: Atlantic forest, conservation, floristic, restoration, threatened species

RESUMO - (Flora arbórea e arbustiva no entorno do Parque Nacional do Iguaçu, PR, Brasil: contribuição para restauração ecológica). Conduzimos um estudo florístico em fragmentos de floresta nativa de uma área localizada entre o Parque Nacional do Iguaçu e a Área de Preservação Permanente do Lago de Itaipu, oeste do Estado do Paraná, objetivando ampliar o conhecimento florístico regional e compilar informações sobre as espécies para subsidiar projetos de restauração naquela área. Encontramos 204 espécies e 51 famílias, sendo as mais ricas Fabaceae (29 spp.), Myrtaceae (18 spp.), Solanaceae (10 spp.), Euphorbiaceae, Meliaceae e Rutaceae (nove spp. cada). A síndrome zoocórica foi a mais comum (69\% das espécies), seguida pela anemocórica (17\%) e autocórica (14\%). As espécies não pioneiras foram as mais frequentes (64\%). Qualitativamente, de modo geral, esses resultados coincidem com o observado em florestas estacionais semideciduais. Com base em dados fitossociológicos de outros estudos, indicamos 34 espécies localmente abundantes que podem ser usadas em maiores proporções em projetos de restauração. Registramos sete espécies ameaçadas de extinção e 15 exóticas, das quais 12 possuem potencial invasivo demandando ações de controle.

Palavras-chave: conservação, espécies ameaçadas, florística, Mata Atlântica, restauração

\section{Introduction}

The Iguaçu National Park (INP) is located in the Alto Paraná Atlantic Forest Ecoregion, the largest among the 15 ecoregions identified in the Atlantic Forest biome (Di Bitetti et al. 2003). It covered originally $471,204 \mathrm{~km}^{2}$ extending from the western side of the Atlantic mountain range, in Brazil, to eastern Paraguay and northeastern Argentina. Its predominant vegetation type is the seasonal semideciduous forest
(Di Bitetti et al. 2003). The vegetation cover in this Ecoregion was reduced to $7.8 \%$ of its original area, the greatest reduction being observed in Brazil where forest cover declined to only $2.7 \%$ of its original area; followed by Paraguay and Argentina, with $13.4 \%$ and $50 \%$ of remaining vegetation, respectively (Di Bitetti et al. 2003). According to these authors, Argentina preserves the largest continuous forest cover in the Alto Paraná Forest Ecoregion, comprising much of the Misiones Province. In the border region between

1. Instituto Florestal, Floresta Estadual de Avaré, Rua Pernambuco, s/n, 18701-180 Avaré, SP, Brasil

2. Instituto Florestal, Divisão de Dasonomia, Rua do Horto, 931, 02377-000 São Paulo, SP, Brasil

3. Universidade Tecnológica Federal do Paraná, Campus Medianeira, Avenida Brasil, 4232, 85884-000 Medianeira, PR, Brasil

4. Corresponding author: cielofbr@yahoo.com.br 
Brazil and Argentina, the Iguaçu National Park stands out as the largest protected area covering a territory of 185,262 ha (Di Bitetti et al. 2003). The native forest cover in this region constitutes the largest continuous remnant of seasonal semideciduous forest in the context of the Atlantic forest biome (Galindo-Leal \& Câmara 2005).

The functional connection between the INP and other protected areas in Argentina and Paraguay as well as in the Brazilian States of Paraná, Santa Catarina, Rio Grande do Sul, São Paulo and Mato Grosso do Sul represents a highly favorable condition for the conservation of the Atlantic Forest biodiversity (Di Bitetti et al. 2003, Galindo-Leal \& Câmara 2005). To this end, some proposals have been discussed, such as the Biodiversity Corridor of the Paraná River (Limont et al. 2015) and the Biodiversity Conservation Landscape for the Alto Paraná Forest Ecoregion (Di Bitetti et al. 2003). These are considered long-term strategies for biodiversity conservation through the maintenance of the ecological process of the biota (Di Bitetti et al. 2003).

An important region for the implementation of those strategies is located between the INP and the southern limit of the permanent preservation area of the Itaipu Lake (PPAIL), in western Paraná State, where the connection between the northern and southern sectors of the Biodiversity Conservation Landscape for the Alto Paraná Forest Ecoregion could be established (Di Bitetti et al. 2003). The PPAIL consists of a 2,900 km long strip, with an average width of $217 \mathrm{~m}$ of reforestation or native forest remnants that runs along almost the entire length of the Itaipu Hydroelectric reservoir margins (Itaipu Binacional 2016a).

However, the connection between the INP and the PPAIL is hindered by the eminently agricultural land use in the region and by the busy BR 277 Highway, which separates apart these two protected areas. The map of the Biodiversity Conservation Landscape for the Alto Paraná Forest Ecoregion indicates the establishment of a biological corridor in this region (Di Bitetti et al. 2003). An example of a biological corridor already established there is the Santa Maria corridor consisting of remnants of native vegetation and a $60-\mathrm{m}$ width reforestation strip in the Bonito River Basin (Itaipu Binacional 2016b). Notwithstanding, a wider biological corridor should yet be designed in the region in order to warrant an effective connection between the two sectors of the
Biodiversity Conservation Landscape for the Alto Paraná Forest Ecoregion (Di Bitetti et al. 2003). Furthermore, the simple recovery of native forests in the river margins and other permanent preservation areas in small rural properties can greatly enhance the functional connectivity at the landscape scale in the region (Seganfredo 2015).

Either for the establishment of a biological corridor between the INP and the PPAIL, or for the recovery of permanent preservation areas in the region, there will be the necessity of forest restoration (sensu Aronson et al. 2011). Floristic knowledge about native vegetation remnants is critical to the choice of the most suitable species for restoration projects (e.g. Monge 2009); to avoid the use of exotic species, and enable high diversity forest restoration. The current floristic knowledge in the region covers the surroundings of the Santa Maria biological corridor, in the Santa Terezinha de Itaipu municipality (Gris 2012), at an average altitude of $270 \mathrm{~m}$ above sea level; and specific areas inside the INP, at altitudes ranging from 150 to $750 \mathrm{~m}$ above sea level (Souza 2015).

The present contribution aimed to extend the regional floristic knowledge in the region between the INP and the PPAIL to a $440 \mathrm{~km}^{2}$ study area covering an altitudinal range expanding from $200 \mathrm{~m}$ to $600 \mathrm{~m}$ above sea level in the municipalities of Céu Azul, Matelândia, Medianeira and Serranópolis do Iguaçu. Beyond a floristic list, we aimed to provide useful information compiled from the literature for the application of the list in restoration projects in the study area, such as successional category, dispersal syndrome, conservation status and indication of locally abundant species.

\section{Material and methods}

Study area - The study area covers predominantly the municipality of Matelândia and, to a lesser extent, of Céu Azul, Medianeira and Serranópolis do Iguaçu, in Paraná State, Brazil. For methodological convenience, we defined the study area as the polygon circumscribed by the Highways BR 277 and PR 495 and by the northwestern border of the INP, covering an area of $440 \mathrm{~km}^{2}$ (figure 1). The average annual temperature in the region is $21.6^{\circ} \mathrm{C}$, with average annual rainfall of $1,803 \mathrm{~mm}$. Climate is subtropical, Cfa according to Köppen classification system (EMBRAPA 2011). Vegetation in the native forest fragments is seasonal semideciduous forest (Di Bitetti et al. 2003) due to the predominance of phanerophytes, the subtropical 


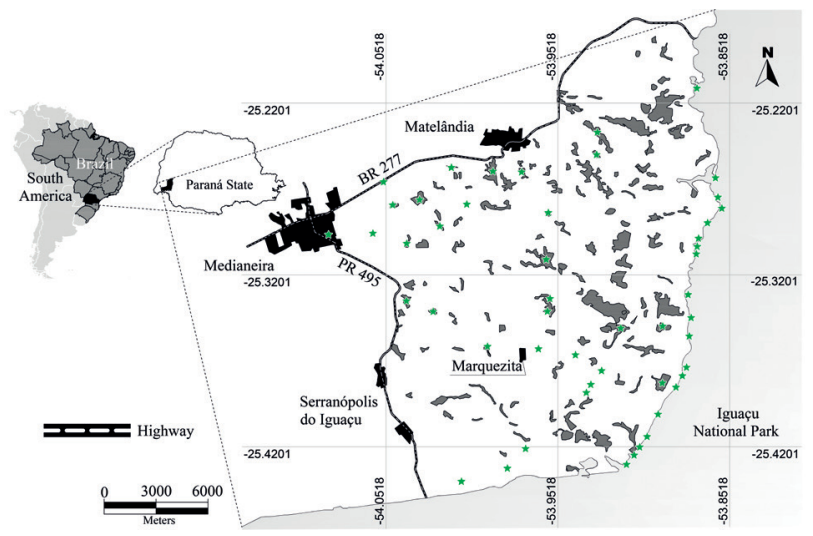

Figure 1. Study area, western Paraná State, Brazil. The area consists of a polygon delimitated by BR 277 and PR 495 highways, and by the northwestern limit of the Iguaçu National Park. The localities of botanical records are indicated by stars. Source: Fundação SOS Mata Atlântica and INPE (2016).

climate seasonality and the leaf shedding of part of the tree individuals (Instituto Brasileiro de Geografia e Estatística 2012). Seasonal semideciduous forests of localities inside the INP, near the study area and extending through a similar altitudinal range, were classified as typical and humid submontane forests by Souza (2015).

Floristic survey - We visited 16 native forest fragments located in the study area from October 2013 to December 2015 during field campaigns that had an average duration of 3 days and monthly periodicity. The number of visits to each fragment along the study period varied, being greater in larger and less disturbed ones. As our objective was to describe the floristic composition of the study area as a whole and not of each fragment individually, we did not compiled an individual floristic list of each fragment, so frequency of species across fragments in the area is not available. Fragments were chosen taking into account the sampling of the entire study area with a distribution of sampling effort as homogeneous as possible, and also considering the access authorization by landowners. We also sampled the northwestern edge of the Iguaçu National Park between the limits of the Park and the so called Old Guarapuava Road between Serranópolis do Iguaçu and Céu Azul municipalities, walking the length of this road in that stretch. The forest fragments studied have different disturbance histories and include secondary forests and primary forests with different levels of degradation (degraded primary forest sensu Aronson et al. 2011). The vegetation of the northwestern edge of the INP was considered as secondary forest. The method used for the floristic survey was based on the walking method (Filgueiras et al. 1994), considering only the tree and shrub components and using trails already existent in the forests. The botanical material was collected with the use of a telescopic pruner and all collections were georeferenced using Global Positioning System (GPS). In addition to the forest fragments and the INP edge, we also collected botanical material along the rural roads in the study area.

We processed the botanical material according to the conventional techniques (Fidalgo \& Bononi 1989). Both fertile and vegetative materials were identified by comparison with herbarium specimens deposited in the Forestry Institute of São Paulo State (Dom Bento José Pickel Herbarium - SPSF), and by consulting the specialized literature. The voucher herbarium sheets were deposited in the SPSF Herbarium, with duplicates deposited in the herbarium of the Federal Technological University of Paraná - Campus Medianeira (Figueira Herbarium - FIG). Only fertile materials were deposited in the SPSF herbarium, while 27 vegetative materials, corresponding to 27 species, were deposited in the FIG herbarium. These species were not found in reproductive phase during the study period, but we decided to incorporate the respective voucher to the FIG herbarium considering the absence of herbarium sheets of these species in this recently established collection (Cielo-Filho et al. 2016).

We sorted the species into families and genera according to the APG IV system (APG IV 2016). To check spelling, corrected and accepted names we consulted the List of Species of the Brazilian Flora (Brazilian Flora 2020 in construction 2016). For most species, plant habits classification was obtained in the List of Species of the Brazilian Flora, but we follow Souza et al. (2013) for palm and fern, although we counted these habits as tree in the results. Verification of endangered species was based on the Red List of Endangered Species of the International Union for Conservation of Nature (International Union for Conservation of Nature 2016) and the Official List of Endangered Species of the Brazilian Flora (Brasil 2014), which was based on the list presented by Martinelli \& Moraes (2013). We did not use the Red List of Paraná State (Paraná 1995), since this list is out of date. With the aim of supporting future forest restoration projects and the establishment of a biological corridor in the study area, we compiled information concerning the successional category (sensu Swaine \& Whitmore 1988) and dispersal 
syndrome (sensu Pijl 1982) of the identified species from the literature (e.g. Lorenzi 1992, Gandolfi et al. 1995, Lorenzi 1998, Silva \& Soares-Silva 2000, Carvalho 2003, Carpanezzi \& Carpanezzi 2006, Carvalho 2006, Carvalho 2008, Lorenzi 2009, Carvalho 2010, Barbosa et al. 2015). We also indicated the native species found in the present contribution which were also registered, as locally abundant species, in phytosociological studies available for the INP and its surroundings (Gris 2012, Souza 2015). To do this, we considered species occupying the first ten positions in the importance value ranking of a typical submontane forest in the Santa Maria Natural Patrimony Private Reservoir (Gris 2012), and of typical and humid submontane forests in the interior of the INP (Souza 2015).

\section{Results}

We performed 940 botanical records of native species, 303 in primary forest and 520 in secondary forest, averaging 51 records per fragment. The remainder 117 records were done on the margins of rural roads. We identified 204 tree and shrub native species from 51 families and 134 genera (table 1). The arboreal habit was represented by 174 species, while the shrubby habit by 96 species. Sixty six species presented both habits. The richest families were Fabaceae (29 species), Myrtaceae (18), Solanaceae (10), Euphorbiaceae, Meliaceae and Rutaceae (nine species each). The richest genera were Eugenia (eight species), Solanum and Zanthoxylum (six species each), Ocotea, Trichilia and Aegiphila (four species each). The most common dispersal syndrome was zoochoric (141 species, 69\%) followed by anemocoric (35 species, 17\%) and autochoric (28 species, 14\%) syndromes. Most (130) species (64\%) belonged to the non-pioneer successional category and the 74 remainder (36\%) was classified as pioneer. Of the 204 recorded species, seven are threatened due to habitat loss and overexploitation (table 2). Additionally to the native species, we found 15 exotic species, of which eight were recorded inside forest fragments and seven in more anthropogenic environments such as roadsides, gardens and pastures (table 3 ).

In table 1 we indicated 14 species reported as locally abundant in Typical Submontane Forest and 10 species reported as locally abundant in Humid Submontane Forest, according to phytosociological studies available for the INP and its surroundings (Gris 2012, Souza 2015). Of the mentioned locally abundant species, only four are pioneer ones: Alchornea triplinervia, Dendropanax cuneatus, Sebastiania brasiliensis and Luehea divaricata.

\section{Discussion}

An important aspect of the present floristic survey was the apparently low species richness, when compared to other studies conducted in Atlantic forests at lower latitudes. For instance, surveys of tree and shrub flora in the seasonal semideciduous forest, carried out in protected areas in the municipalities of Paranapanema and Itapeva, both in São Paulo State, registered 349 and 317 species, respectively (CieloFilho et al. 2009, Souza et al. 2012). However, as pointed out by these authors, the high richness in those areas may be related to the presence of Cerrado remnants in the region, which would expand the regional species pool, increasing the richness in remnants of seasonal semideciduous forest. Even without the influence of the Cerrado flora, the number of tree and shrub species (266) reported by Rossetto \& Vieira (2013) for the seasonal semideciduous forest of the Mata dos Godoy State Park, northern Paraná State, was higher than that found in the present study.

It could be suggested that the flora in northern remnants of seasonal semideciduous forest of the Paraná State is richer than in the remnants located at higher latitudes of the State, due to more rigorous climatic conditions. For instance, the number of tree species (197) reported for the Mata dos Godoy State Park, where a comprehensive floristic study in a well conserved forest remnant was carried out (Rossetto \& Vieira 2013), was greater than that found in our study (174), as well as in the studies of Souza (2015) and Gris (2012) which identified, respectively, 165 and 112 native tree species in the INP and forest fragments nearby. However, when considered together, the floristic data available for the study area and its surroundings - this study, Gris (2012) and Souza (2015) - summed up 228 native tree species, what suggests that the tree flora in northern remnants of seasonal semideciduous forest of the Paraná State is not richer than in southern ones.

Another aspect to be considered in these richness comparisons is the differences concerning methods and anthropogenic disturbance histories among study areas. For instance, the use of an inclusion criterion to sampling plant individuals - stem perimeter at breast height equal or more than $15 \mathrm{~cm}$ - by Gris (2012) and Souza (2015) may have limited the number of species 
Table 1. Tree and shrub native flora in western Paraná State, Brazil. SD, dispersal syndrome: AUT: autochoric, ANE: anemochoric, ZOO: zoochoric; CS, successional category: P: pioneer, NP: non-pioneer; sh: shrub, tr: tree; FIG and SPSF, record number in the FIG and SPSF herbaria. C, complementary information: species reported as locally abundant in typical (a) and humid (b) forests in the Iguaçu National Park and surroundings (Gris 2012, Souza 2015), locally abundant pioneer species according to visual assessment (c) and tree species exclusive of the present study (e).

\begin{tabular}{llllllll}
\hline Family/Species & Vernacular name & Habit & SD & CS & FIG & SPSF & C \\
\hline
\end{tabular}

Pteridophyta

Cyatheaceae

Alsophila sternbergii (Sternb.) D.S. Conant

Gimnospermae

Araucariaceae

Araucaria angustifolia (Bertol.) Kuntze

Angiospermae

Acanthaceae

Aphelandra schottiana (Nees) Profice

Ruellia angustiflora (Ness) Lindau ex Rambo

Ruellia cf. brevifolia (Pohl) C. Ezcurra

Anacardiaceae

Astronium graveolens Jacq.

Schinus terebinthifolius Raddi

Annonaceae

Annona emarginata (Schltdl.) H.Rainer

Apocynaceae

Aspidosperma australe Müll.Arg.

Aspidosperma polyneuron Müll.Arg.

Rauwolfia sellowii Müll.Arg.

Tabernaemontana catharinensis A.DC.

Tabernaemontana hystrix Steud.

Aquifoliaceae

Ilex paraguariensis A.St.-Hil.

Araliaceae

Aralia warmingiana (Marchal) J. Wen

Dendropanax cuneatus (DC.) Decne.

$\&$ Planch.

Schefflera morototoni (Aubl.) Maguire et al.

Arecaceae

Euterpe edulis Mart.

Syagrus romanzoffiana (Cham.) Glassman

Asteraceae

Baccharis dracunculifolia DC.

Dasyphyllum brasiliense (Spreng.) Cabrera

Piptocarpha rotundifolia (Less.) Baker

Bignoniaceae

Handroanthus albus (Cham.) Mattos

Handroanthus heptaphyllus (Vell.) Mattos samambaiaçu

fern

ANE

araucária

$\mathrm{ZOO}$

NP

$235 \quad 48974$

anil-bravo

guiné

pingo-de-sangue

guaritá

aroeira-vermelha

tr ANE

sh, tr ZOO

NP

53650099

araticum-mirim

sh, $\operatorname{tr} \quad \mathrm{ZOO}$

P

259

49005

$$
\begin{gathered}
\text { pequiá } \\
\text { peroba-rosa } \\
\text { casca-d'anta } \\
\text { jasmin } \\
\text { leiteiro }
\end{gathered}
$$

erva-mate

carobão

maria-mole

mandioqueiro

palmito-juçara

jeriva

vassourinha

sucará

candeia

ipê-amarelo-da-serra

ipê-roxo tr ANE

tr ANE

tr $\mathrm{ZOO}$

sh, tr ZOO

tr $\mathrm{ZOO}$

NP

257

49003

NP $204 \quad 48865$

NP $\quad 65 \quad 48211$

$\begin{array}{lll}P & 243 & 48962\end{array}$

P $\quad 256 \quad 49002$

sh, tr ZOO NP $296 \quad 49174$

$\operatorname{tr}$

$\mathrm{ZOO}$

$\mathrm{P}$

585

$\operatorname{tr}$

ZOO

$\mathrm{P}$

167

48628

a

ZOO P 598

palm

NP

308

$49189 \quad a$

palm ZOO

443

49528 a,b

sh ANE P

477

49559

sh, tr ANE

NP 586

606

e

tr

ANE

NP

e

a c c
ZOO 
Table 1 (continuation)

\begin{tabular}{|c|c|c|c|c|c|c|c|}
\hline Family/Species & Vernacular name & Habit & SD & $\mathrm{CS}$ & FIG & SPSF & $\mathrm{C}$ \\
\hline Handroanthus ochraceus (Cham.) Mattos & ipê-amarelo & $\operatorname{tr}$ & ANE & NP & 237 & 48993 & \\
\hline Jacaranda micrantha Cham. & caroba-miúda & $\operatorname{tr}$ & ANE & $\mathrm{P}$ & 207 & 48860 & \\
\hline \multicolumn{8}{|l|}{ Boraginaceae } \\
\hline Cordia americana (L.) Gottschling \& J.S. Mill. & guaiuvira & $\operatorname{tr}$ & ANE & NP & 317 & 49198 & $\mathrm{~b}$ \\
\hline Cordia ecalyculata Vell. & louro-mole & $\operatorname{tr}$ & $\mathrm{ZOO}$ & NP & 51 & 48129 & \\
\hline Cordia trichotoma (Vell.) Arrab. ex Steud. & louro-pardo & $\operatorname{tr}$ & ANE & NP & 91 & 48324 & \\
\hline Heliotropium transalpinum Vell. & pau-de-sapo & $\mathrm{sh}$ & $\mathrm{ZOO}$ & $\mathrm{P}$ & 250 & 48996 & \\
\hline \multicolumn{8}{|l|}{ Cannabaceae } \\
\hline Celtis ehrenbergiana (Klotzsch) Liebm. & esporão-de-galo & sh, tr & $\mathrm{ZOO}$ & NP & 513 & 50081 & e \\
\hline Celtis iguanaea (Jacq.) Sarg. & gumbixava & sh, tr & $\mathrm{ZOO}$ & NP & 106 & 48320 & \\
\hline Celtis spinosa Spreng. & grão-de-galo & $\operatorname{sh}$ & $\mathrm{ZOO}$ & $\mathrm{P}$ & 261 & 49007 & \\
\hline Trema micrantha (L.) Blume & candiúba & sh, tr & $\mathrm{ZOO}$ & $\mathrm{P}$ & 348 & 49273 & $\mathrm{c}$ \\
\hline \multicolumn{8}{|l|}{ Cardiopteridaceae } \\
\hline Citronella paniculata (Mart.) R.A. Howard & falsa-congonheira & $\operatorname{tr}$ & $\mathrm{ZOO}$ & NP & 617 & & \\
\hline \multicolumn{8}{|l|}{ Caricaceae } \\
\hline Jacaratia spinosa (Aubl.) A. DC. & jaracatiá & $\operatorname{tr}$ & $\mathrm{ZOO}$ & NP & 281 & 49159 & \\
\hline \multicolumn{8}{|l|}{ Celastraceae } \\
\hline Maytenus aquifolia Mart. & falsa-espinheira-santa & sh, tr & $\mathrm{ZOO}$ & NP & 607 & & \\
\hline \multicolumn{8}{|l|}{ Clusiaceae } \\
\hline Garcinia gardneriana (Planch. \& Triana) Zappi & bacupari & sh, $\operatorname{tr}$ & $\mathrm{ZOO}$ & NP & 269 & 49016 & \\
\hline \multicolumn{8}{|l|}{ Ebenaceae } \\
\hline Diospyros inconstans Jacq. & marmelinho & $\mathrm{sh}, \operatorname{tr}$ & $\mathrm{ZOO}$ & NP & 603 & & e \\
\hline \multicolumn{8}{|l|}{ Erythroxylaceae } \\
\hline Erythroxylum argentinum O.E. Schulz & cocão & sh, tr & $\mathrm{ZOO}$ & NP & 386 & 49300 & $\mathrm{e}$ \\
\hline Erythroxylum cuneifolium (Mart.) O.E. Schulz & fruta-de-pomba & $\mathrm{sh}$ & $\mathrm{ZOO}$ & NP & 486 & 49639 & \\
\hline Erythroxylum deciduum A.St.-Hil. & marmelinho & $\mathrm{sh}, \mathrm{tr}$ & $\mathrm{ZOO}$ & NP & 377 & 49284 & \\
\hline \multicolumn{8}{|l|}{ Euphorbiaceae } \\
\hline Acalypha amblyodonta (Müll.Arg.) Müll. Arg. & tapa-buraco & $\mathrm{sh}$ & AUT & $\mathrm{P}$ & 508 & 49786 & \\
\hline Alchornea glandulosa Poepp. \& Endl. & tanheiro, tapiá & sh, tr & $\mathrm{ZOO}$ & $\mathrm{P}$ & 124 & 48633 & \\
\hline Alchornea triplinervia (Spreng.) Müll.Arg. & pau-jangada & sh, tr & $\mathrm{ZOO}$ & $\mathrm{P}$ & 23 & 47898 & $\mathrm{a}$ \\
\hline Bernardia pulchella (Baill.) Müll.Arg. & canela-de-virá & $\mathrm{sh}$ & AUT & $\mathrm{P}$ & 395 & 49304 & \\
\hline Croton triqueter Lam. & velane & $\mathrm{sh}$ & AUT & $\mathrm{P}$ & 506 & 49787 & \\
\hline Croton urucurana Baill. & sangra-d'água & $\operatorname{tr}$ & AUT & $\mathrm{P}$ & 385 & 49257 & $\mathrm{e}$ \\
\hline Gymnanthes klotzschiana Müll.Arg. & branquilho & sh, tr & AUT & NP & 404 & 49334 & \\
\hline Sapium glandulosum (L.) Morong & pau-de-leite & sh, tr & $\mathrm{ZOO}$ & $\mathrm{P}$ & 602 & & e \\
\hline Sebastiania brasiliensis Spreng. & branquilho & sh, tr & AUT & $\mathrm{P}$ & 4 & 47908 & $\mathrm{~b}$ \\
\hline \multicolumn{8}{|l|}{ Fabaceae } \\
\hline $\begin{array}{l}\text { Albizia cf. edwallii (Hoehne) Barneby \& } \\
\text { J.W. Grimes }\end{array}$ & gurujuba & $\mathrm{sh}$ & AUT & NP & 396 & 49301 & \\
\hline Albizia niopoides (Benth.) Burkart & farinha-seca & $\operatorname{tr}$ & AUT & $\mathrm{P}$ & 599 & & \\
\hline Anadenanthera colubrina (Vell.) Brenan & angico-branco & sh, tr & AUT & NP & 406 & 49337 & \\
\hline Apuleia leiocarpa (Vogel.) J.F. Macbr. & garapa & $\operatorname{tr}$ & ANE & NP & 376 & 49248 & \\
\hline
\end{tabular}


Table 1 (continuation)

\begin{tabular}{|c|c|c|c|c|c|c|c|}
\hline Family/Species & Vernacular name & Habit & SD & $\mathrm{CS}$ & FIG & SPSF & $\mathrm{C}$ \\
\hline Bauhinia longifolia (Bong.) Steud. & pata-de-vaca & sh, tr & AUT & $\mathrm{P}$ & 35 & 48229 & $\mathrm{e}$ \\
\hline Bauhinia forficata Link & unha-de-vaca & $\operatorname{tr}$ & AUT & $\mathrm{P}$ & 399 & 49292 & \\
\hline Calliandra foliolosa Benth. & cabelo-de-anjo & sh, tr & AUT & NP & 253 & 48999 & \\
\hline Dalbergia frutescens (Vell.) Britton & rabo-de-bugio & $\mathrm{sh}$ & ANE & NP & 14 & 47894 & \\
\hline Enterolobium contortisiliquum (Vell.) Morong & tamboril & $\operatorname{tr}$ & $\mathrm{ZOO}$ & $\mathrm{P}$ & 173 & 48894 & \\
\hline Erythrina falcata Benth. & corticeira-da-serra & $\operatorname{tr}$ & AUT & NP & 231 & 48977 & \\
\hline Holocalyx balansae Micheli & alecrim & $\operatorname{tr}$ & $\mathrm{ZOO}$ & NP & 135 & 48648 & \\
\hline Inga marginata Willd. & ingá-feijão & $\operatorname{tr}$ & $\mathrm{ZOO}$ & NP & 3 & 47906 & \\
\hline Inga vera Willd. & ingá-banana & $\operatorname{tr}$ & $\mathrm{ZOO}$ & $\mathrm{P}$ & 39 & 48232 & \\
\hline $\begin{array}{l}\text { Lonchocarpus cultratus (Vell.) A.M.G. Azevedo } \\
\text { \& H.C. Lima }\end{array}$ & embira-de-sapo & $\operatorname{tr}$ & ANE & $\mathrm{P}$ & 20 & 47896 & \\
\hline Machaerium hirtum (Vell.) Stelfelld & jacarandá-de-espinho & $\operatorname{tr}$ & ANE & NP & 478 & 49565 & e \\
\hline Machaerium paraguariense Hassl. & sapuvão & $\operatorname{tr}$ & ANE & NP & 41 & 48231 & \\
\hline Machaerium stipitatum Vogel & sapuva & $\operatorname{tr}$ & ANE & NP & 90 & 48341 & $a, b$ \\
\hline Mimosa bimucronata (DC.) Kuntze & maricá & sh, tr & AUT & $\mathrm{P}$ & 490 & 49770 & \\
\hline $\begin{array}{l}\text { Muellera campestris (Mart. ex Benth.) M.J. } \\
\text { Silva \& A.M.G.Azevedo }\end{array}$ & embirinha & $\operatorname{tr}$ & ANE & NP & 390 & 49302 & \\
\hline Myrocarpus frondosus Allemão & cabreúva-amarela & $\operatorname{tr}$ & ANE & NP & 294 & 49172 & \\
\hline Parapiptadenia rigida (Benth.) Brenan & angico-da-mata & $\operatorname{tr}$ & AUT & NP & 113 & 48677 & \\
\hline Peltophorum dubium (Spreng.) Taub. & canafístula & $\operatorname{tr}$ & ANE & $\mathrm{P}$ & 12 & 47916 & $\mathrm{c}$ \\
\hline Pterocarpus rohrii Vahl & pau-sangue & $\operatorname{tr}$ & ANE & NP & 601 & & e \\
\hline Pterogyne nitens Tul. & amendoim & $\operatorname{tr}$ & ANE & NP & 597 & & \\
\hline Senegalia polyphylla (DC.) Britton \& Rose & monjoleiro & sh, tr & AUT & $\mathrm{P}$ & 81 & 48225 & \\
\hline Senegalia tenuifolia (L.) Britton \& Rose & serra-goela & $\mathrm{sh}$ & AUT & $\mathrm{P}$ & 448 & 49536 & \\
\hline Senna alata (L.) Roxb. & mata-pasto & sh, tr & AUT & $P$ & 600 & & e \\
\hline $\begin{array}{l}\text { Senna macranthera (DC. ex Collad.) H.S. } \\
\text { Irwin \& Barneby }\end{array}$ & manduirana & sh, tr & AUT & $P$ & 182 & 48884 & e \\
\hline $\begin{array}{l}\text { Senna pendula (Humb.\& Bonpl.ex } \\
\text { Willd.) H.S.Irwin \& Barneby }\end{array}$ & canudo-de-pito & sh, tr & AUT & $\mathrm{P}$ & 183 & 48872 & e \\
\hline \multicolumn{8}{|l|}{ Lamiaceae } \\
\hline Aegiphila brachiata Vell. & tamanqueiro & sh, tr & $\mathrm{ZOO}$ & $\mathrm{P}$ & 378 & 49293 & e \\
\hline Aegiphila integrifolia (Jacq.) Moldenke & caiuia, gaioleira & sh, tr & $\mathrm{ZOO}$ & $\mathrm{P}$ & 382 & 49290 & \\
\hline Aegiphila mediterranea Vell. & pau-de-tamanco & $\operatorname{tr}$ & $\mathrm{ZOO}$ & $\mathrm{P}$ & 71 & 48221 & \\
\hline Aegiphila novofriburgensis Moldenke & tamanqueiro & sh & $\mathrm{ZOO}$ & $\mathrm{P}$ & 34 & 48226 & \\
\hline Vitex megapotamica (Spreng.) Moldenke & tarumã & sh, tr & $\mathrm{ZOO}$ & NP & 389 & 49282 & \\
\hline \multicolumn{8}{|l|}{ Lauraceae } \\
\hline Nectandra lanceolata Nees & canela amarela & $\operatorname{tr}$ & $\mathrm{ZOO}$ & NP & 7 & 47904 & \\
\hline Nectandra megapotamica (Spreng.) Mez & canelinha & $\operatorname{tr}$ & $\mathrm{ZOO}$ & NP & 29 & 48137 & $a, b$ \\
\hline Ocotea diospyrifolia (Meisn.) Mez & canela-louro & $\operatorname{tr}$ & $\mathrm{ZOO}$ & NP & 306 & 49185 & $a, b$ \\
\hline Ocotea puberula (Rich.) Nees & canela-guaicá & $\operatorname{tr}$ & $\mathrm{ZOO}$ & NP & 116 & 48676 & \\
\hline Ocotea pulchella (Nees \& Mart.) Mez & canela-lajeana & $\operatorname{tr}$ & $\mathrm{ZOO}$ & NP & 596 & & $\mathrm{e}$ \\
\hline Ocotea silvestris Vattimo-Gil & canela-do-campo & $\operatorname{tr}$ & $\mathrm{ZOO}$ & NP & 60 & 48135 & \\
\hline
\end{tabular}


Table 1 (continuation)

\begin{tabular}{|c|c|c|c|c|c|c|c|}
\hline Family/Species & Vernacular name & Habit & SD & $\mathrm{CS}$ & FIG & SPSF & $\mathrm{C}$ \\
\hline \multicolumn{8}{|l|}{ Laxmanniaceae } \\
\hline Cordyline spectabilis Kunth \& Bouché & guaraiva & $\operatorname{tr}$ & $\mathrm{ZOO}$ & $\mathrm{P}$ & 616 & & \\
\hline \multicolumn{8}{|l|}{ Loganiaceae } \\
\hline Strychnos brasiliensis Mart. & salta-martim & $\operatorname{sh}$ & $\mathrm{ZOO}$ & NP & 408 & 49327 & \\
\hline \multicolumn{8}{|l|}{ Malvaceae } \\
\hline Bastardiopsis densiflora (Hook. \& Arn.) Hassl. & jangada-brava & $\operatorname{tr}$ & ANE & $\mathrm{P}$ & 153 & 48629 & $\mathrm{c}$ \\
\hline Ceiba speciosa (A.St.-Hil.) Ravenna & paineira & $\operatorname{tr}$ & ANE & NP & 105 & 48335 & \\
\hline Guazuma ulmifolia Lam. & mutamba-preta & $\operatorname{tr}$ & $\mathrm{ZOO}$ & $\mathrm{P}$ & 109 & 48654 & \\
\hline Heliocarpus popayanensis Kunth & jangada-brava & $\operatorname{tr}$ & ANE & $\mathrm{P}$ & 175 & 48891 & $\mathrm{c}$ \\
\hline Luehea divaricata Mart. \& Zucc. & açoita-cavalo & $\operatorname{tr}$ & ANE & $\mathrm{P}$ & 57 & 48113 & $\mathrm{~b}$ \\
\hline Pavonia sepium A.St.-Hil. & carrapicho & sh, tr & AUT & NP & 504 & 49782 & $\mathrm{e}$ \\
\hline Sida cf. planicaulis Cav. & guanxuma & $\operatorname{sh}$ & AUT & $\mathrm{P}$ & 566 & 50128 & \\
\hline \multicolumn{8}{|l|}{ Melastomataceae } \\
\hline Miconia cinerascens Miq. & jacatirão & sh, tr & $\mathrm{ZOO}$ & $\mathrm{P}$ & 507 & 49784 & $\mathrm{e}$ \\
\hline Miconia discolor DC. & apaga-brasa & $\operatorname{tr}$ & $\mathrm{ZOO}$ & NP & 380 & 49305 & $\mathrm{e}$ \\
\hline Miconia pusilliflora (DC.) Naudin & pixirica & sh, tr & $\mathrm{ZOO}$ & $\mathrm{P}$ & 89 & 48322 & \\
\hline \multicolumn{8}{|l|}{ Meliaceae } \\
\hline Cabralea canjerana (Vell.) Mart. & canjerana & $\operatorname{tr}$ & $\mathrm{ZOO}$ & NP & 150 & 48673 & a \\
\hline Cedrela fissilis Vell. & cedro & $\operatorname{tr}$ & ANE & NP & 40 & 48128 & $\mathrm{a}$ \\
\hline Guarea guidonia (L.) Sleumer & marinheiro & sh, tr & $\mathrm{ZOO}$ & NP & 588 & & \\
\hline Guarea kunthiana A. Juss. & canjambo & $\operatorname{tr}$ & $\mathrm{ZOO}$ & NP & 5 & 47914 & $\mathrm{a}$ \\
\hline Guarea macrophylla Vahl & baga-de-morcego & $\operatorname{tr}$ & $\mathrm{ZOO}$ & NP & 101 & 48326 & \\
\hline Trichilia catigua A. Juss. & catiguá & $\operatorname{tr}$ & $\mathrm{ZOO}$ & NP & 273 & 49020 & \\
\hline Trichilia clausseni C. DC. & quebra-machado & $\operatorname{tr}$ & $\mathrm{ZOO}$ & NP & 224 & 48992 & \\
\hline Trichilia elegans A. Juss. & catiguazinho & sh, tr & $\mathrm{ZOO}$ & NP & 169 & 48632 & \\
\hline Trichilia pallida Sw. & baga-de-morcego & $\operatorname{tr}$ & $\mathrm{ZOO}$ & NP & 202 & 48873 & \\
\hline \multicolumn{8}{|l|}{ Monimiaceae } \\
\hline Hennecartia omphalandra J. Poiss. & canema & $\operatorname{tr}$ & $\mathrm{ZOO}$ & NP & 614 & & \\
\hline \multicolumn{8}{|l|}{ Moraceae } \\
\hline Ficus guaranitica Chodat & figueira-branca & $\operatorname{tr}$ & $\mathrm{ZOO}$ & NP & 26 & 47900 & $\mathrm{e}$ \\
\hline Ficus insipida Willd. & figueira-mata-pau & $\operatorname{tr}$ & $\mathrm{ZOO}$ & $\mathrm{P}$ & 1 & 47919 & \\
\hline Ficus luschnathiana (Miq.) Miq. & figueira & $\operatorname{tr}$ & $\mathrm{ZOO}$ & NP & 367 & 48960 & \\
\hline Maclura tinctoria (L.) D.Don ex Steud. & taiuva & $\operatorname{tr}$ & $\mathrm{ZOO}$ & NP & 18 & 47913 & \\
\hline Sorocea bonplandii (Baill.) W.C. Burger et al. & cincho & sh, tr & $\mathrm{ZOO}$ & NP & 227 & 48978 & $\mathrm{a}$ \\
\hline \multicolumn{8}{|l|}{ Myrtaceae } \\
\hline Calyptranthes concinna DC. & guamirim-facho & $\operatorname{tr}$ & $\mathrm{ZOO}$ & NP & 466 & 49550 & \\
\hline Campomanesia guaviroba (DC.) Kiaersk. & gabiroba & $\operatorname{tr}$ & $\mathrm{ZOO}$ & NP & 277 & 49154 & $\mathrm{e}$ \\
\hline $\begin{array}{l}\text { Campomanesia guazumifolia (Cambess.) } \\
\text { O. Berg }\end{array}$ & sete-capotes & $\operatorname{tr}$ & $\mathrm{ZOO}$ & NP & 371 & 49211 & \\
\hline Campomanesia xanthocarpa (Mart.) O. Berg & gabiroba & $\operatorname{tr}$ & $\mathrm{ZOO}$ & NP & 333 & 49266 & \\
\hline Eugenia burkartiana (D. Legrand) D. Legrand & guamirim & $\operatorname{tr}$ & $\mathrm{ZOO}$ & NP & 286 & 49164 & \\
\hline Eugenia florida DC. & guamirim & $\operatorname{tr}$ & $\mathrm{ZOO}$ & NP & 613 & & \\
\hline
\end{tabular}


Table 1 (continuation)

\begin{tabular}{|c|c|c|c|c|c|c|c|}
\hline Family/Species & Vernacular name & Habit & SD & CS & FIG & SPSF & $\mathrm{C}$ \\
\hline Eugenia hiemalis Cambess. & guamirim-miúdo & sh, tr & $\mathrm{ZOO}$ & $\mathrm{P}$ & 123 & 48656 & \\
\hline Eugenia involucrata DC. & cereja-do-rio-grande & $\operatorname{tr}$ & $\mathrm{ZOO}$ & NP & 297 & 49175 & \\
\hline Eugenia pyriformis Cambess. & uvaia & sh, tr & $\mathrm{ZOO}$ & $\mathrm{P}$ & 46 & 48133 & $\mathrm{c}$ \\
\hline Eugenia ramboi D. Legrand & batinga-branca & $\operatorname{tr}$ & $\mathrm{ZOO}$ & NP & 587 & & \\
\hline Eugenia subterminalis DC. & cambuí-pitanga & $\operatorname{tr}$ & $\mathrm{ZOO}$ & NP & 61 & 48132 & \\
\hline Eugenia uniflora L. & pitangueira & sh, tr & $\mathrm{ZOO}$ & NP & 334 & 49260 & e \\
\hline Myrceugenia euosma (O. Berg) D. Legrand & cambuizinho & $\operatorname{tr}$ & $\mathrm{ZOO}$ & $\mathrm{P}$ & 591 & & e \\
\hline Myrcia pulchra (O. Berg) Kiaersk. & guamirim & $\operatorname{tr}$ & $\mathrm{ZOO}$ & NP & 592 & & $\mathrm{e}$ \\
\hline Myrcia splendens (Sw.) DC. & jambinho, guamirim & $\operatorname{tr}$ & $\mathrm{ZOO}$ & NP & 593 & & e \\
\hline Myrcianthes pungens (O. Berg) D. Legrand & guabiju & $\operatorname{tr}$ & $\mathrm{ZOO}$ & NP & 16 & 47901 & $\mathrm{e}$ \\
\hline Myrciaria tenella (DC.) O. Berg & cambuí & $\operatorname{tr}$ & $\mathrm{ZOO}$ & NP & 594 & & $\mathrm{e}$ \\
\hline Plinia rivularis (Cambess.) Rotman & guapuriti, piúna & $\operatorname{tr}$ & $\mathrm{ZOO}$ & NP & 206 & 48869 & \\
\hline \multicolumn{8}{|l|}{ Nyctaginaceae } \\
\hline Guapira hirsuta (Choisy) Lundell & maria-mole & sh, tr & ANE & NP & 447 & 49531 & e \\
\hline Guapira opposita (Vell.) Reitz & flor-de-pérola & sh, tr & $\mathrm{ZOO}$ & NP & 67 & 48213 & e \\
\hline Neea pendulina Heimerl & maria-mole & sh, tr & $\mathrm{ZOO}$ & $\mathrm{P}$ & 595 & & \\
\hline Pisonia ambigua Heimerl & maria-faceira & $\operatorname{tr}$ & $\mathrm{ZOO}$ & NP & 78 & 48222 & \\
\hline \multicolumn{8}{|l|}{ Phyllanthaceae } \\
\hline Margaritaria nobilis L.f. & sobragirana & sh, tr & $\mathrm{ZOO}$ & NP & 50 & 48131 & $\mathrm{e}$ \\
\hline \multicolumn{8}{|l|}{ Phytolaccaceae } \\
\hline Phytolacca dioica $\mathrm{L}$. & cebolão & $\operatorname{tr}$ & $\mathrm{ZOO}$ & $\mathrm{P}$ & 230 & 48959 & \\
\hline \multicolumn{8}{|l|}{ Piperaceae } \\
\hline Piper aduncum L. & pimenta longa & sh, tr & $\mathrm{ZOO}$ & $\mathrm{P}$ & 107 & 48328 & $\mathrm{c}, \mathrm{e}$ \\
\hline Piper amalago L. & jaborandi & $\mathrm{sh}$ & $\mathrm{ZOO}$ & NP & 108 & 48329 & \\
\hline \multicolumn{8}{|l|}{ Polygonaceae } \\
\hline Ruprechtia laxiflora Meisn. & marmeleiro-do-mato & $\operatorname{tr}$ & ANE & NP & 295 & 49173 & \\
\hline \multicolumn{8}{|l|}{ Primulaceae } \\
\hline Geissanthus ambiguus (Mart.) G. Agostini & pau-de-charco & sh & $\mathrm{ZOO}$ & NP & 610 & & \\
\hline Myrsine coriacea (Sw.) R.Br. ex Roem. \& Schult. & capororoca & sh, tr & $\mathrm{ZOO}$ & $\mathrm{P}$ & 302 & 49180 & $\mathrm{c}$ \\
\hline Myrsine loefgrenii (Mez) Imkhan. & pororoca & $\operatorname{tr}$ & $\mathrm{ZOO}$ & NP & 270 & 49017 & e \\
\hline Myrsine umbellata Mart. & copororoca & $\operatorname{tr}$ & $\mathrm{ZOO}$ & NP & 44 & 48127 & \\
\hline \multicolumn{8}{|l|}{ Proteaceae } \\
\hline $\begin{array}{l}\text { Roupala montana var. brasiliensis } \\
\text { (Klotzsch) K.S.Edwards }\end{array}$ & carvalho-brasileiro & sh, tr & ANE & NP & 266 & 49012 & \\
\hline \multicolumn{8}{|l|}{ Rosaceae } \\
\hline Prunus myrtifolia (L.) Urb. & pessegueiro-bravo & $\operatorname{tr}$ & $\mathrm{ZOO}$ & NP & 2 & 47905 & \\
\hline Rubus erythroclados Mart. ex Hook. f. & amora-branca & sh & $\mathrm{ZOO}$ & $\mathrm{P}$ & 473 & 49566 & \\
\hline \multicolumn{8}{|l|}{ Rubiaceae } \\
\hline Coussarea contracta (Walp.) Müll.Arg. & pasto-de-anta & sh, tr & $\mathrm{ZOO}$ & NP & 260 & 49006 & e \\
\hline Ixora venulosa Benth. & ixora-do-mato & sh, tr & $\mathrm{ZOO}$ & NP & 590 & & \\
\hline Palicourea marcgravii A.St.-Hill & erva-do-rato & $\operatorname{sh}$ & $\mathrm{ZOO}$ & NP & 364 & 49184 & \\
\hline Psychotria carthagenensis Jacq. & erva-de-gralha & sh, tr & $\mathrm{ZOO}$ & NP & 239 & 48968 & \\
\hline
\end{tabular}


Table 1 (continuation)

\begin{tabular}{|c|c|c|c|c|c|c|c|}
\hline Family/Species & Vernacular name & Habit & SD & $\mathrm{CS}$ & FIG & SPSF & $\mathrm{C}$ \\
\hline Psychotria leiocarpa Cham. \& Schltdl. & grandiúva-de-anta & $\mathrm{sh}$ & $\mathrm{ZOO}$ & NP & 405 & 49332 & \\
\hline Randia armata (Sw.) DC. & laranja-de-macaco & sh, tr & $\mathrm{ZOO}$ & NP & 418 & 49352 & $\mathrm{e}$ \\
\hline \multicolumn{8}{|l|}{ Rutaceae } \\
\hline Balfourodendron riedelianum (Engl.) Engl. & pau-marfim & $\operatorname{tr}$ & ANE & NP & 32 & 48118 & $a, b$ \\
\hline Helietta apiculata Benth. & canela-de-veado & $\operatorname{tr}$ & ANE & NP & 431 & 49349 & \\
\hline Pilocarpus pennatifolius Lem. & jaborandi & $\operatorname{tr}$ & AUT & NP & 103 & 48333 & \\
\hline Zanthoxylum caribaeum Lam. & arruda-brava & $\operatorname{tr}$ & $\mathrm{ZOO}$ & NP & 216 & 48958 & \\
\hline Zanthoxylum fagara (L.) Sarg. & tembetari, mamica & sh, tr & $\mathrm{ZOO}$ & NP & 163 & 48627 & e \\
\hline Zanthoxylum monogynum A.St.-Hil. & Juvá & $\operatorname{tr}$ & $\mathrm{ZOO}$ & NP & 618 & & e \\
\hline Zanthoxylum petiolare A.St.-Hil. \& Tul. & mamica-de-porca & $\operatorname{tr}$ & $\mathrm{ZOO}$ & NP & 441 & 49533 & \\
\hline Zanthoxylum rhoifolium Lam. & mamica-de-cadela & $\operatorname{tr}$ & $\mathrm{ZOO}$ & NP & 357 & 49251 & \\
\hline Zanthoxylum riedelianum Engl. & mamica-de-porca & $\operatorname{tr}$ & $\mathrm{ZOO}$ & NP & 247 & 48957 & $\mathrm{e}$ \\
\hline \multicolumn{8}{|l|}{ Salicaceae } \\
\hline Banara tomentosa Clos & cambroé & sh, tr & $\mathrm{ZOO}$ & NP & 329 & 49524 & \\
\hline Casearia decandra Jacq. & cafezeiro-do-mato & sh, tr & $\mathrm{ZOO}$ & NP & 328 & 49155 & \\
\hline Casearia gossypiosperma Briq. & espeteiro & $\operatorname{tr}$ & $\mathrm{ZOO}$ & $\mathrm{P}$ & 190 & 48880 & \\
\hline Casearia sylvestris $\mathrm{Sw}$. & carvalinho & sh, tr & $\mathrm{ZOO}$ & $\mathrm{P}$ & 203 & 48867 & $\mathrm{c}$ \\
\hline Prockia crucis P. Browne ex L. & cuiteleiro & sh, tr & $\mathrm{ZOO}$ & NP & 303 & 49181 & \\
\hline Xylosma ciliatifolia (Clos) Eichler & espinho-de-agulha & sh, tr & $\mathrm{ZOO}$ & NP & 565 & 50127 & \\
\hline \multicolumn{8}{|l|}{ Sapindaceae } \\
\hline $\begin{array}{l}\text { Allophylus edulis (A.St.-Hil. et al.) Hieron. } \\
\text { ex Niederl. }\end{array}$ & chal-chal, vacum & sh, tr & $\mathrm{ZOO}$ & $\mathrm{P}$ & 242 & 48965 & $\mathrm{c}$ \\
\hline Cupania vernalis Cambess. & camboatã-vermelho & $\operatorname{tr}$ & $\mathrm{ZOO}$ & NP & 154 & 48626 & \\
\hline Diatenopteryx sorbifolia Radlk. & maria-preta & $\operatorname{tr}$ & ANE & $\mathrm{P}$ & 264 & 49010 & c \\
\hline Matayba elaeagnoides Radlk. & camboatã-branco & sh, tr & $\mathrm{ZOO}$ & NP & 331 & 49263 & \\
\hline \multicolumn{8}{|l|}{ Sapotaceae } \\
\hline $\begin{array}{l}\text { Chrysophyllum gonocarpum (Mart. \& Eichler } \\
\text { ex Miq.) Engl. }\end{array}$ & caxeta-amarela & $\operatorname{tr}$ & $\mathrm{ZOO}$ & NP & 15 & 47912 & $a, b$ \\
\hline $\begin{array}{l}\text { Chrysophyllum marginatum (Hook. \& } \\
\text { Arn.) Radlk. }\end{array}$ & aguaí & $\operatorname{tr}$ & $\mathrm{ZOO}$ & NP & 58 & 48108 & \\
\hline \multicolumn{8}{|l|}{ Simaroubaceae } \\
\hline Picrasma crenata (Vell.) Engl. & pau-amargo & $\operatorname{tr}$ & $\mathrm{ZOO}$ & NP & 358 & 49000 & \\
\hline \multicolumn{8}{|l|}{ Solanaceae } \\
\hline Acnistus arborescens (L.) Schltdl. & marianeira & sh & $\mathrm{ZOO}$ & $\mathrm{P}$ & 361 & 49250 & \\
\hline $\begin{array}{l}\text { Aureliana wettsteiniana (Witasek) Hunz. } \\
\text { \& Barboza }\end{array}$ & fumeirinho & $\operatorname{tr}$ & $\mathrm{ZOO}$ & $\mathrm{P}$ & 387 & 49298 & e \\
\hline Cestrum intermedium Sendtn. & coreana & sh, tr & $\mathrm{ZOO}$ & $\mathrm{P}$ & 238 & 48961 & \\
\hline Cestrum strigilatum Ruiz \& Pav. & coerana & sh, tr & $\mathrm{ZOO}$ & $\mathrm{P}$ & 104 & 48325 & \\
\hline Solanum asperolanatum Ruiz \& Pav. & jurubeba & $\operatorname{sh}$ & $\mathrm{ZOO}$ & $\mathrm{P}$ & 453 & 49542 & \\
\hline Solanum caavurana Vell. & caavurana & sh & $\mathrm{ZOO}$ & $\mathrm{P}$ & 345 & 49269 & $\mathrm{c}$ \\
\hline Solanum mauritianum Scop. & fona-de-porco & sh, tr & $\mathrm{ZOO}$ & NP & 350 & 49280 & \\
\hline Solanum pseudoquina A. St.-Hil. & tabaqueira & $\operatorname{tr}$ & $\mathrm{ZOO}$ & $\mathrm{P}$ & 433 & 49428 & \\
\hline Solanum sanctae-catharinae Dunal & joá-manso & $\operatorname{tr}$ & $\mathrm{ZOO}$ & $\mathrm{P}$ & 485 & 49638 & \\
\hline
\end{tabular}


Table 1 (continuation)

\begin{tabular}{|c|c|c|c|c|c|c|c|}
\hline Family/Species & Vernacular name & Habit & SD & $\mathrm{CS}$ & FIG & SPSF & $\mathrm{C}$ \\
\hline Solanum variabile Mart. & jurubeba & sh, tr & $\mathrm{ZOO}$ & NP & 369 & 49208 & $\mathrm{e}$ \\
\hline \multicolumn{8}{|l|}{ Styracaceae } \\
\hline Styrax leprosus Hook. \& Arn. & carne-de-vaca & $\operatorname{tr}$ & $\mathrm{ZOO}$ & $\mathrm{P}$ & 346 & 49432 & $\mathrm{c}$ \\
\hline \multicolumn{8}{|l|}{ Symplocaceae } \\
\hline Symplocos celastrinea Mart. & mate-falso & sh, tr & $\mathrm{ZOO}$ & NP & 492 & 49767 & $\mathrm{e}$ \\
\hline Symplocos estrellensis Casar. & canela-conserva & sh, tr & $\mathrm{ZOO}$ & NP & 474 & 49557 & e \\
\hline \multicolumn{8}{|l|}{ Urticaceae } \\
\hline Boehmeria caudata Sw. & urtiga-mansa & sh & $\mathrm{ZOO}$ & NP & 241 & 48987 & \\
\hline Cecropia pachystachya Trécul & embaúba & $\operatorname{tr}$ & $\mathrm{ZOO}$ & $\mathrm{P}$ & 25 & 47899 & $\mathrm{c}$ \\
\hline Urera baccifera (L.) Gaudich. ex Wedd. & urtiga & sh, tr & $\mathrm{ZOO}$ & NP & 319 & 49200 & $\mathrm{~b}$ \\
\hline \multicolumn{8}{|l|}{ Verbenaceae } \\
\hline Aloysia gratissima (Gillies \& Hook.) Tronc. & cidrilha & sh & $\mathrm{ZOO}$ & $\mathrm{P}$ & 502 & 49789 & \\
\hline Aloysia virgata (Ruiz \& Pav.) Juss. & cambará-de-lixa & sh, tr & $\mathrm{ZOO}$ & $\mathrm{P}$ & 99 & 48332 & $\mathrm{c}$ \\
\hline Citharexylum solanaceum Cham. & pau-viola & $\operatorname{tr}$ & $\mathrm{ZOO}$ & $\mathrm{P}$ & 355 & 49281 & $\mathrm{e}$ \\
\hline Lantana camara $\mathrm{L}$. & camará & sh, tr & $\mathrm{ZOO}$ & $\mathrm{P}$ & 85 & 48115 & $\mathrm{e}$ \\
\hline Lantana trifolia $\mathrm{L}$ & lantana & $\mathrm{sh}$ & $\mathrm{ZOO}$ & NP & 461 & 49549 & \\
\hline Stachytarpheta cayennensis (Rich.) Vahl & rincão & $\mathrm{sh}$ & $\mathrm{ZOO}$ & $\mathrm{P}$ & 366 & 49151 & \\
\hline \multicolumn{8}{|l|}{ Violaceae } \\
\hline Pombalia bigibbosa (A.St.-Hil.) Paula-Souza & ganha-saia & sh & AUT & $\mathrm{P}$ & 82 & 48114 & $\mathrm{c}$ \\
\hline Pombalia communis (A.St.-Hil.) Paula-Souza & apanha-saia & sh & AUT & NP & 438 & 49430 & \\
\hline
\end{tabular}

recorded in those studies. This kind of methodological limitation may also have restricted the tree species richness found in other studies, such as in the Rio das Cobras Farm, southwestern Paraná State - 128 species (Viani et al. 2011); in protected areas of the Argentine Province of Misiones: Iguazú National Park and its surroundings - 72 species (Chediack 2008) and Osununú Private Reserve - 96 species (Velazco et al. 2015); in protected areas of the Paraguayan side of the area of influence of the Itaipu Lake - 127 species (Monge 2009); and in the São Francisco State Park, northern Paraná State, where 113 tree species were recorded, although in this case the relatively low richness seems to be also related to the intensive practice of selective logging before the protected area had been established (Tomé et al. 1999, Zama et al. 2012).

It is worth to note that 43 native tree species recorded in the present floristic survey (table 1) were not registered by Gris (2012) and Souza (2015) in the INP and its surroundings. This may be a consequence of sampling effect, but also of floristic particularities of the study area in relation to its surroundings. Of that 43 species, $70 \%$ are non-pioneer, $72 \%$ have zoochoric dispersal syndrome, while the other two dispersal syndromes comprised each, $14 \%$ of the remainder species. Comparing these data with the corresponding percentages observed for the whole set of species in this study, there is no evidence of bias concerning successional category or dispersal syndromes for the set of species exclusive of the present research. However, in relation to the distribution of exclusive species among families, there was a highlight for Myrtaceae, with seven of the 18 species found being exclusive. This may be a consequence of the relatively complex taxonomy of this family. Another highlight may be perceived for the distribution of species between habits: $56 \%$ of the exclusive species present both arboreal and shrubby habit, while the corresponding value for the whole set of species in this study is of $32 \%$. We suggest that because we did not use an inclusion criterion to sampling plant individuals as the other studies referred above - stem perimeter at breast height equal or more than $15 \mathrm{~cm}$ - we attained a better representation of the flora composed by species presenting simultaneously the arboreal and shrubby habits. Furthermore, the overrepresentation of the shrubby-arboreal habit among the exclusive species may be a consequence of the intensive sampling of edge habitats in the present study, as indicated by the 
Table 2. Threatened species recorded in the study area, in western Paraná State, Brazil, according to the lists of the International Union for Conservation of Nature (IUCN) and the Brazilian Ministry of the Environment (MMA). CR, Critically Endangered; EN, Endangered; VU, Vulnerable. The information concerning the type of threat was obtained from Martinelli \& Moraes (2013) and from IUCN (2016).

\begin{tabular}{|c|c|c|c|}
\hline Family/Species & IUCN & MMA & Type of threat \\
\hline \multicolumn{4}{|l|}{ Apocynaceae } \\
\hline Aspidosperma polyneuron & EN & & Timber exploitation. \\
\hline \multicolumn{4}{|l|}{ Araucariaceae } \\
\hline Araucaria angustifolia & $\mathrm{CR}$ & EN & Habitat loss, timber exploitation. \\
\hline \multicolumn{4}{|l|}{ Arecaceae } \\
\hline Euterpe edulis & & VU & Habitat loss, exploitation of the palm heart. \\
\hline \multicolumn{4}{|l|}{ Fabaceae } \\
\hline Apuleia leiocarpa & & VU & Timber exploitation. \\
\hline \multicolumn{4}{|l|}{ Meliaceae } \\
\hline Cedrela fissilis & EN & VU & Habitat loss, timber exploitation. \\
\hline \multicolumn{4}{|l|}{ Myrtaceae } \\
\hline Myrcianthes pungens & $\mathrm{EN}$ & & Habitat loss. \\
\hline \multicolumn{4}{|l|}{ Rutaceae } \\
\hline Baufourodendron riedelianum & EN & & Habitat loss, timber exploitation. \\
\hline
\end{tabular}

Table 3. Exotic species recorded in the study area, western Paraná State, Brazil. F, species recorded inside forest fragments; A, species recorded in anthropogenic environments such as roadsides, gardens and pastures.

\begin{tabular}{lr}
\hline Family/Species & $\mathrm{F}$ \\
\hline Anacardiaceae & $\mathrm{X}$ \\
Mangifera indica L. &
\end{tabular}

Asteraceae

Gymnanthemum amygdalinum (Delile) Sch.Bip. ex Walp.

Bignoniaceae

Handroanthus impetiginosus (Mart. ex DC.) Mattos

Tabebuia roseoalba (Ridl.) Sandwith

Tecoma stans (L.) Juss. ex Kunth

Bixaceae

Bixa orellana $\mathrm{L}$.

F

Fabaceae

Leucaena leucocephala (Lam.) de Wit

$\mathrm{X}$

Lauraceae

Persea americana Mill.

$\mathrm{X}$

Magnoliaceae

Michelia champaca $\mathrm{L}$.

$\mathrm{X}$

Meliaceae

Melia azedarach L.

$\mathrm{X}$

Myrtaceae

Psidium guajava $\mathrm{L}$.

Syzygium jambos (L.) Alston
$\mathrm{X}$

$\mathrm{X}$

A

$\mathrm{X}$

$\mathrm{X}$

X

$\mathrm{X}$

$\mathrm{X}$

continue 
Table 3 (continuation)

\begin{tabular}{|c|c|c|}
\hline \multirow{2}{*}{\multicolumn{3}{|c|}{$\begin{array}{l}\text { Family/Species } \\
\text { Rhamnaceae }\end{array}$}} \\
\hline & & \\
\hline Hovenia dulcis Thunb. & $\mathrm{X}$ & \\
\hline \multicolumn{3}{|l|}{ Rosaceae } \\
\hline Eriobotrya japonica (Thunb.) Lindl. & $\mathrm{X}$ & \\
\hline \multicolumn{3}{|l|}{ Rutaceae } \\
\hline Citrus limon (L.) Osbeck & $\mathrm{X}$ & \\
\hline
\end{tabular}

greater percentage of shrub-tree-pioneer species in the set exclusive species $(9 / 43=21 \%)$ than in the whole set of species $(29 / 204=14 \%)$. Among the exclusive species, there was one threatened, Myrcianthes pungens, found in both primary and secondary forest fragments in the study area, what stress the importance of these remnants to biodiversity conservation.

On the other hand, 54 native tree species were registered by Gris (2012) and Souza (2015), but not by the present study. The data set resulting from the results of those authors complies study sites at altitudes ranging from 150 to $750 \mathrm{~m}$ above sea level, while the present study area covers an altitudinal range expanding from $200 \mathrm{~m}$ to $600 \mathrm{~m}$ above sea level, and $68 \%$ of our botanical records were done in study sites located at altitudes between $300 \mathrm{~m}$ and $500 \mathrm{~m}$. The weak representation of the regional altitudinal extremes in our data set may help to explain the absence in our species list of the 54 species exclusive of the lists reported by Gris (2012) and Souza (2015). Another possible explanation comes from the landscape context: our study sites are situated in a highly fragmented landscape in which some species may have been lost because of edge effect, dispersal barriers and other process associated with forest fragmentation leading to local species extinction (Primack \& Rodrigues 2001).

The distribution of species richness among families in the study area was similar to that found in other seasonal semideciduous forest studies in the Brazilian States of São Paulo (Cielo-Filho et al. 2009, Souza et al. 2012) and Paraná (Viani et al. 2011, Gris 2012, Zama et al. 2012, Rossetto \& Vieira 2013); in the Province of Misiones, Argentina (Chediack 2008, Velazco et al. 2015); and in the Departments of Canindeyú and Alto Paraná, Paraguay (Monge 2009). However, an interesting feature of the study area was the relatively low representativeness of Lauraceae and the relatively high richness of Solanaceae. This could suggest a particularity of the flora of seasonal semideciduous forest remnants located in higher latitudes of the Paraná State, but, instead, seems to be a consequence of forest fragmentation or sampling effect, as discussed above. For instance, we recorded only six species of Lauraceae, but this number increases to 10 considering the results of floristic surveys nearby (Gris 2012, Souza 2015), equaling the number found in the Mata dos Godoy State Park in northern Paraná (Rossetto \& Vieira 2013). In relation to Solanaceae, the number of tree and shrub species found in the Mata dos Godoy State Park, 19, was even higher than that found in the study area and its surroundings, 15 (this study, Gris 2012, Souza 2015). The presence of Fabaceae and Myrtaceae in the first two richness ranking positions is a characteristic shared by other studies of the tree and shrub flora in seasonal semideciduous forests of Argentina, Brazil and Paraguay (Cielo-Filho et al. 2009, Monge 2009, Souza et al. 2012, Rossetto \& Vieira 2013, Velazco et al. 2015).

Despite the above considerations about an apparent lack of particularities in the flora of the study area concerning species richness and families representativeness, a botanist familiarized with the flora of seasonal semideciduous forest remnants located at lower latitudes that came to visit forest remnants in the study area and its surroundings, will still encounter some peculiarities of the tree and shrub flora of these higher latitudes remnants concerning species composition. For instance, she/he will miss some notorious species like, among others: Croton floribundus Spreng, Copaifera langsdorffii Desf., Esenbeckia febrifuga (A.St.-Hil.) A.Juss. ex Mart., Gallesia integrifolia (Spreng.) Harms, Machaerium nyctitans (Vell.) Benth., Piptadenia gonoacantha (Mart.) J.F.Macbr., Zeyheria tuberculosa (Vell.) Bureau ex Verl., Geonoma schottiana Mart. (and Arecaceae in general), Moquiniastrum polymorphum (Less.) G. Sancho (and Asteraceae in general), Miconia theizans (Bonpl.) Cogn. (and Melastomataceae in general). 
It must be stressed that some species found by Gris (2012) in plantings done in the Permanent Preservation Area of Itaipu Lake and in the reforestation strip of the Santa Maria biological corridor may not be native of the region. We highlight the following exotic or probably exotic species registered in Gris (2012), but not in the present contribution nor in Souza (2015): Annona neosericea H. Rainer, Handroanthus impetiginosus Mattos, Croton floribundus, Manihot grahamii Hook., Anadenanthera peregrina (L.) Speg., Calliandra brevipes Benth., Cassia leptophylla Vogel, Copaifera langsdorffi, Machaerium nyctitans, Piptadenia gonoacantha, Poincianella pluviosa (DC.) L.P. Queiroz. and Eugenia gracillima Kiaersk.. Together with the species explicitly stated as exotic of the Brazilian territory in Gris (2012), these species should be avoided in future restoration projects in the region.

Corroborating the ranking of representativeness of dispersal syndromes observed in other studies carried out in seasonal semideciduous forest (Silva \& Soares-Silva 2000, Santos \& Kinoshita 2003, Kinoshita et al. 2006, Zama et al. 2012) and agreeing with the general pattern observed in tropical forests (Howe \& Smallwood 1982), the most frequent dispersal syndrome was the zoochory $(69 \%)$, followed by anemochory (17\%) and autochory (14\%). The percentage of zoochoric species that we found in this study was higher than that found in other studies in well preserved seasonal semideciduous forests (Silva \& Soares-Silva 2000, Santos \& Kinoshita 2003, Kinoshita et al. 2006), what shows a good overall representativeness of zoochoric species in our floristic sampling. The same can be inferred for the representativeness of non-pioneer species, $64 \%$, a percentage higher than that found in the Mata dos Godoy State Park, where $55.8 \%$ of the species found may be considered as non-pioneers, taking the late secondary and climax categories adopted by Silva \& Soares-Silva (2000) together. The Mata dos Godoy State Park shelters a well preserved seasonal semideciduous forest remnant in advanced successional stage (Silva \& Soares-Silva 2000, Rossetto \& Vieira 2013). Given the importance of a good representativeness of non-pioneer and zoochoric species in restoration projects, our study presents a promising species list to support initiatives of this nature in the area studied. In line with this assertion is the inclusion of the shrubby habit in the present floristic survey, since the use of non-tree species in restoration projects have been recently encouraged (Durigan et al. 2010, Barbosa et al. 2015).
The endangered species recorded in this study denote the overall conservation status of the Atlantic forest, especially with regard to the drastic reduction in vegetation cover (Ribeiro et al. 2009) resulting in significant habitat loss. Another aspect that stands out is the overexploitation of natural resources (timber and palm heart in the case of Euterpe edulis). The most critically threatened species we found in the study area was Araucaria angustifolia, considered "critically endangered" and "endangered", respectively, in the IUCN and MMA consulted lists. It should be noted that this species is rare in the study area, occurring mostly in anthropogenic sectors situated on the edge of the INP, in the strip sampled at the margins of the old Guarapuava road. This could suggest that those specimens were planted there by the first settlers who arrived in the area in the 1950s and 1960s. On the other hand, the presence of adult reproductive individuals of Araucaria angustifolia at the beginning of the colonization period was confirmed by ancient settlers in neighborhood of the Marquezita town, but not for other localities in the study area (N.L. Viapiana, personal communication), indicating that the species is native, but was not widespread in that area. In the highest elevations of the INP, above the altitudinal range of our study area, Souza (2015) identified a transitional zone between seasonal semideciduous forest and ombrophilous mixed forest, where Araucaria angustifolia is one of the most abundant species. Additionally, Ombrophilous Mixed Forests are also encountered not so far away from our study area (less than $150 \mathrm{~km}$ ), for instance, in the Argentine Province of Misiones (e.g. in the Cruce Caballero Provincial Park (Rios 2006)), and in vast tracts of the Southwest region of Paraná State (Castella \& Britez 2004). Notwithstanding, as this species is locally rare and not widespread in our study area, we did not recommend its extensive use in restoration projects in this area. On the other hand, we encouraged the widespread use in such projects of all the other endangered species registered in the present study.

The locally abundant species indicated in table 1 are, in general, more prone to become well established in restoration areas and, so, their use in higher proportions can favor the rapid forest recover. In using the indication of locally abundant species in table 1 to design restoration projects, one should consider if the species were reported as locally abundant in typical or humid submontane forest, and apply this last species category in the restoration of sites located in river margins and depressions of the terrain, where 
soils are more humid, while the first species category must be reserved to the restoration of sites with drier soil conditions. Some of the locally abundant species can be applied in both situations (table 1). Another consideration refers to the successional category of the locally abundant species indicated, the majority of which, non-pioneer. As restoration plantings, in general, apply a greater proportion of pioneer than non-pioneer species, it would be interesting the indication of additional locally abundant pioneer species, besides the four already mentioned. To do this, we listed the following pioneer species considered as locally abundant through visual assessment by the authors (table 1): Tabernaemontana catharinensis, Trema micrantha, Peltophorum dubium, Bastardiopsis densiflora, Heliocarpus popayanensis, Eugenia pyriformis, Piper aduncum, Myrsine coriacea, Casearia sylvestris, Allophylus edulis, Diatenopteryx sorbifolia, Solanum caavurana, Styrax leprosus, Cecropia pachystachya, Aloysia virgata and Pombalia bigibbosa. Naturally, all species listed in table 1 should be considered in restoration plantings in the study area, the ones not cited as locally abundant should be considered for the achievement of high diversity plantings.

Among the exotic species found in the study area, with the exception of Michelia champaca, Gymnanthemum amygdalinum and Syzygium jambos, all others have confirmed invasive potential, especially Tecoma stans, Leucaena leucocephala and Hovenia dulcis (Zenni \& Ziller 2011). Such species constitute serious threats to biodiversity conservation in the region and should be targeted by control actions aimed at the eradication of their populations in natural environments. Additionally, actions aiming to prevent anthropogenic disturbances in the forest remnants should be implemented in order to allows secondary forests in the study area to reach more advanced successional stages, since the susceptibility to invasion by exotic species may be greater in forests of earlier successional stages, as suggested for the invasion by Hovenia dulcis in forests of the upper Uruguay region (Lazzarin et al. 2015).

In conclusion, we reported the floristic composition of an area situated in a strategic region for the conservation of the seasonal semideciduous forest in the context of the Atlantic forest biome. In general, regarding species richness, we did not detect any particularity of the flora of the study area and its surroundings in relation to the flora of seasonal semideciduous forest remnants located at lower latitudes. The same can be said regarding species distribution among families, and frequency distribution of dispersal syndromes and ecological groups, for the flora of the study area. However, some peculiarities emerged due to the lack in the study area of certain tree species commonly found in those remnants. We provided information on dispersal syndromes and successional categories for 204 tree and shrub species and indicated 34 locally abundant and seven threatened ones, in order to aid in the design of restoration projects in the study area. We also identified 15 exotic tree species in the area, of which, 12 have invasive potential, requiring eradication programs.

\section{Acknowledgments}

We are grateful to $\mathrm{CNPq}$ for the scientific initiation scholarship granted to Carolina Rodrigues Sousa and Juliana Menezes de Jesus; to the UTFPR, Medianeira Campus, to the Forestry Institute of São Paulo State, to Itaipu Binacional, to Chico Mendes Institute of Biodiversity Conservation and to the Department of Environment of Medianeira and Matelândia municipalities; to the several undergraduate students and other volunteers who helped in the collection and processing of botanical material; to the Herbarium technician Mr. Ernane Lino da Silva; to the landowners who allowed the entrance into their properties, especially to Mr. Ângelo Baratto, Mr. José Nilson de Oliveira, Ms. Luciane Trauczynski dos Santos, Ms. Mariza Ângela Biazuz and Mr. Odair Camargo.

\section{Literature cited}

Aronson, J., Durigan, G. \& Brancalion, P.H.S. 2011. Conceitos e definições correlatos à ciência e à prática da restauração ecológica. IF Série Registros 44: 1-38.

Angiosperm Phylogeny Group - APG. 2016. An update of the Angiosperm Phylogeny Group classification for the orders and families of flowering plants: APG IV. Botanical Journal of the Linnean Society. 181:1-20.

Barbosa, L.M., Shirasuna, R.T., Lima, F.C. \& Ortiz, P.R.T. 2015. Lista de espécies indicadas para restauração ecológica para diversas regiões do estado de São Paulo. In: VI Simpósio de Restauração Ecológica: Novos Rumos e Perspectivas, 2015, São Paulo: Imprensa Oficial do Estado (Imesp).

Brasil. 2014. Ministério do Meio Ambiente. Lista Nacional Oficial de Espécies da Flora Ameaçadas de Extinção. Portaria n. 443, de 17 de dezembro de 2014. Diário Oficial da União. 18 de dezembro, Seção I, pp. 110-121. 
Brazilian Flora 2020 (in construction). 2016. Rio de Janeiro Botanical Garden. Available in http:// floradobrasil.jbrj.gov.br/ (access in 16-IV-2016).

Carpanezzi, A.A. \& Carpanezzi, O.T. 2006. Espécies Nativas Recomendadas para Recuperação Ambiental no Estado do Paraná em Solos não Degradados. Embrapa Florestas, Colombo (PR).

Carvalho, P.E.R. 2003. Espécies arbóreas brasileiras. Embrapa, Brasília. v.1.

Carvalho, P.E.R. 2006. Espécies arbóreas brasileiras. Embrapa, Brasília. v.2.

Carvalho, P.E.R. 2008. Espécies arbóreas brasileiras. Embrapa, Brasília. v.3.

Carvalho, P.E.R. 2010. Espécies arbóreas brasileiras. Embrapa, Brasília. v.4.

Castella, P.R., Britez, R.M. 2004. A floresta com araucária no Paraná: conservação e diagnóstico dos remanescentes florestais. Ministério do Meio Ambiente, Brasília (DF).

Chediack, S. 2008. Efecto de la explotación forestal sobre la estructura, diversidad y composición florística de los palmitales de la Selva Atlántica en Misiones, Argentina. Revista de Biología Tropical 56: 721-728.

Cielo-Filho, R., Baitello, J.B., Pastore, J.A., Aguiar, O.T., Souza, S.C.P.M., Toniato, M.T.Z., Lima, C.R. de \& Ribeiro, A.P. 2009. Ampliando a densidade de coletas botânicas na região da bacia hidrográfica do Alto Paranapanema: Caracterização florística da Floresta Estadual e da Estação Ecológica de Paranapanema. Biota Neotrop. 9: 255-276. Available in http://www.biotaneotropica.org.br/v9n3/en/ abstract?inventory+bn02009032009 (access in 01VIII-2016).

Cielo-Filho, R., Periotto, F., Câmara, C.D., Franco, G.A., Baitello, J.B., Aguiar, O.T., Sousa, C.R. 2016. Cooperação científica para implantação do Herbário da Figueira FIG. Revista do Instituto Florestal 28: 77-81.

Di Bitetti, M.S., Placci, G. \& Dietz, L.A. 2003. Uma visão de Biodiversidade para a Ecorregião Florestas do Alto Paraná - Bioma Mata Atlântica: planejando a paisagem de conservação da biodiversidade e estabelecendo prioridades para ações de conservação. World Wildlife Fund, Washington (DC).

Durigan, G., Engel, V.L., Torezan, J.M., Melo, A.C.G., Marques, M.C.M., Martins, S.V., Reis, A., Scarano, F.R. 2010. Normas jurídicas para a restauração ecológica: uma barreira a mais para dificultar o êxito das iniciativas? Revista Árvore 34: 471-485.

EMBRAPA (Empresa Brasileira de Pesquisa Agropecuária). 2011. Atlas Climático da Região Sul do Brasil: Estados do Paraná, Santa Catarina e Rio Grande do Sul. Embrapa Clima Temperado, Pelotas; Embrapa Florestas, Colombo.

Filgueiras, T.S., Nogueira, P.E., Brochado, A.L., Guala II, G.F. 1994. Caminhamento - um método expedito para levantamentos florísticos qualitativos. Caderno Geoc 12:39-43.
Fidalgo, O. \& Bononi, V.L.R. 1989. Técnicas de coleta, preservação e herborização de material botânico. São Paulo: Instituto de Botânica/Imprensa Oficial do Estado de São Paulo.

INPE (Fundação SOS Mata Atlântica \& Instituto Nacional de Pesquisas Espaciais). 2016. Atlas dos remanescentes florestais da Mata Atlântica, período de 2014-2015. São Paulo, Fundação SOS Mata Atlântica \& INPE, São Jose dos Campos.

Galindo-Leal, C. \& Câmara, I.G. 2005. Status do hotspot Mata Atlântica: uma síntese. In: C. Galindo-Leal, I.G. Câmara (ed.). Mata Atlântica: biodiversidade, ameaças e perspectivas. São Paulo: Fundação SOS Mata Atlântica. pp. 3-11.

Gandolfi, S., Leitão-Filho, H.F. \& Bezerra, C.L.F. 1995. Levantamento florístico e caráter sucessional das espécies arbustivo-arbóreas de uma Floresta Mesófila Semidecídua no município de Guarulhos, SP. Revista Brasileira de Biologia 55: 753-767.

Gris, D. 2012. Riqueza e similaridade da vegetação arbórea do Corredor de Biodiversidade Santa Maria, PR. Dissertação de Mestrado, Universidade Estadual do Oeste do Paraná, UNIOESTE, Cascavel, Paraná.

Howe, H.F. \& Smallwood, J. 1982. Ecology of seed dispersal. Annual Review of Ecology and Systematics, Palo Alto 13:201-228.

IBGE (Instituto Brasileiro de Geografia e Estatística). 2012. Manual técnico da vegetação brasileira: sistema fitogeográfico, inventário das formações florestais e campestres, técnicas e manejo de coleções botânicas, procedimento para mapeamento. IBGE - Coordenação de Recursos Naturais e Estudos Ambientais, 2. ed. revisto e ampliado. Rio de Janeiro.

IUCN (International Union for Conservation of Nature). 2016. The IUCN red list of threatened species. Available in www.iucn.org (access in 16-IV-2016).

Itaipu Binacional. 2016a. Reposição Florestal. Available in http://www.itaipu.gov.br/meioambiente/reposicaoflorestal (access in 14 de abril de 2016).

Itaipu Binacional. 2016b. Corredor de Biodiversidade Santa Maria. Available in http://www.itaipu.gov.br/ meioambiente/corredor-de-biodiversidade (access in 14-IV-2016).

Kinoshita, L.S., Torres, R.B., Forni-Martins, E.R., Spinelli, T., Ahn, Y.J. \& Constâncio, S.S. 2006. Composição florística e síndromes de polinização e de dispersão da mata do Sítio São Francisco, Campinas, SP, Brasil. Acta Botanica Brasilica 20: 313-327.

Lazzarin, L.C., Silva, A.C., Higuchi, P., Souza, K., Perin, J.E. \& Cruz, A.P. 2015. Invasão biológica por Hovenia dulcis Thunb. em fragmentos florestais na região do alto Uruguai, Brasil. Revista Árvore 39: 1007-1017.

Limont, M., Müller, C., Soares, N. (orgs.). 2015. Ações de governança territorial da Rede Gestora do Corredor de Biodiversidade do Rio Paraná. Instituto Curicaca, Porto Alegre. 
Lorenzi, H. 1992. Árvores brasileiras: manual de identificação e cultivo de plantas arbóreas nativas do Brasil. Instituto Plantarum de Estudos da Flora LTDA, Nova Odessa (SP).

Lorenzi, H. 1998. Árvores brasileiras: manual de identificação e cultivo de plantas arbóreas nativas do Brasil. Instituto Plantarum de Estudos da Flora LTDA, Nova Odessa (SP).

Lorenzi, H. 2009. Árvores brasileiras: manual de identificação e cultivo de plantas arbóreas nativas do Brasil. Instituto Plantarum de Estudos da Flora LTDA, Nova Odessa (SP).

Martinelli, G. \& Moraes, M.A. (org.). 2013. Livro vermelho da flora do Brasil. Rio de Janeiro. CNCFLORA, Instituto de Pesquisas Jardim Botânico do Rio de Janeiro.

Monge, V.R.V. 2009. Comparação de associações vegetais sobre diferentes tipos de solo na área de influência da Represa Itaipu, para reconhecimento de espécies apropriadas para restauração ecológica. Tese de Doutorado, Escola Superior de Agricultura Luiz de Queiroz - Universidade de São Paulo, Piracicaba, São Paulo.

Paraná. 1995. Lista Vermelha de Plantas Ameaçadas de Extinção no Estado do Paraná. Secretaria de Estado do Meio Ambiente - SEMA, Curitiba.

Pijl, L.V. 1982. Principles of dispersal in higher plants. Berlim: Springer-Verlag.

Primack, R.B. \& Rodrigues, E. 2001. Biologia da conservação. Londrina. Editora Planta.

Ribeiro, M.C., Metzger, J.P., Martensen, A.C., Ponzoni, F.J. \& Hirota, M.M. 2009. The Brazilian Atlantic Forest: how much is left, and how is the remaining forests distributed? Implications for conservation. Biological Conservation 142: 1141-1153.

Rios, R.C. 2006. Caracterização florística e fitossociológica da vegetação arbórea em três unidades pedológicas do Parque provincial Cruce Caballero, Misiones, Argentina. Dissertação de Mestrado, Universidade Federal do Paraná, Curitiba, Paraná.

Rossetto, E.F.S. \& Vieira, A.O.S. 2013. Vascular Flora of the Mata dos Godoy State Park, Londrina, Paraná, Brazil. CheckList 9: 1020-1034.

Santos, K. \& Kinoshita, L.S. 2003. Flora arbustivo-arbórea do fragmento de floresta estacional semidecidual do Ribeirão Cachoeira, município de Campinas, SP. Acta Botanica Brasilica 17: 325-341.
Seganfredo, D. 2015. Estudo da cobertura vegetal nativa da bacia do rio Ocoy, Oeste do Paraná: subsídios para a implantação de um corredor de biodiversidade entre o Parque Nacional do Iguaçu e o Lago de Itaipu. Dissertação de Mestrado, Universidade Tecnológica Federal do Paraná - Câmpus Medianeira, Medianeira, Paraná.

Silva, F.C. \& Soares-Silva, L.H. 2000. Arboreal flora of the Godoy Forest State Park, Londrina, PR, Brazil. Edinburgh Journal of Botany 57: 107-120.

Sousa, R.F. 2015. Fitossociologia e dinâmica da vegetação arbórea no Parque Nacional do Iguaçu. Dissertação de Mestrado, Universidade Federal do Paraná, Curitiba, Paraná.

Souza, V.C., Flores, T.B., Lorenzi, H. 2013. Introdução à Botânica: Morfologia. Instituto Plantarum de Estudos da Flora LTDA, Nova Odessa (SP).

Souza, S.C.P.M., Cielo-Filho, R., Baitello, J.B., Aguiar, O.T., Pastore, J.A., Franco, G.A.D.C., Toniato, M.T.Z., Barbosa, A.C.O., Lima, C.R., Costa, N.O. \& Pinto, N.S. 2012. Caracterização florística de uma área de contato entre Cerrado e Mata Atlântica na região Sudoeste do Estado de São Paulo. Revista do Instituto Florestal 24: 99-132.

Swaine, M.D. \& Whitmore, T.C. 1988. On the definition of ecological species groups in tropical rain forests. Vegetatio 75: 81-86.

Tomé, M.V.F., Miglioranza, E., Vilhena, A.H. \& Fonseca, E.P. 1999. Composição florística e fitossociológica do Parque Estadual Mata São Francisco. Revista do Instituto Florestal 11: 13-23.

Velazco, S.J.E., Galvão, F., Keller, H.A., Bedrij, N.A. 2015. Florística e fitossociologia de uma Floresta Estacional Semidecidual, Reserva Privada OsununúMisiones, Argentina. Floresta e Ambiente 22: 1-12.

Viani, R.A.G, Costa, J.C., Rozza, A.F., Bufo, L.V.B., Ferreira, M.A.P. \& Oliveira, A.C.P. 2011. Caracterização florística e estrutural de remanescentes florestais de Quedas do Iguaçu, Sudoeste do Paraná. Biota Neotropica 11: 115-128. Available in http://www.biotaneotropica. org.br/v11n1/en/abstract?article+bn01911012011 (01-VIII-2016).

Zama, M.Y., Bovolenta, Y.R., Carvalho, E.S., Rodrigues, D.R., Araujo, C.G., Sorace, M.A.F. \& Luz, D.G. 2012. Florística e síndromes de dispersão de espécies arbustivo-arbóreas no Parque Estadual Mata São Francisco, PR, Brasil. Hoehnea 39: 369-378.

Zenni, R.D. \& Ziller, S.R. 2011. An overview of invasive plants in Brazil. Brazilian Journal of Botany 34: 431-446. 